\title{
Shot noise in gravitational-wave detectors with Fabry-Perot arms
}

\author{
Torrey T. Lyons, Martin W. Regehr, and Frederick J. Raab
}

\begin{abstract}
Shot-noise-limited sensitivity is calculated for gravitational-wave interferometers with Fabry-Perot arms, similar to those being installed at the Laser Interferometer Gravitational-Wave Observatory (LIGO) and the Italian-French Laser Interferometer Collaboration (VIRGO) facility. This calculation includes the effect of nonstationary shot noise that is due to phase modulation of the light. The resulting formula is experimentally verified by a test interferometer with suspended mirrors in the 40-m arms. (C) 2000 Optical Society of America

OCIS codes: $120.3180,030.5260,120.2230,000.2780$.
\end{abstract}

\section{Introduction}

Interferometric gravitational-wave detectors with multiple-kilometer baselines are currently under construction by the Laser Interferometer GravitationalWave Observatory (LIGO) ${ }^{1}$ project in the United States and the Italian-French Laser Interferometer Collaboration (VIRGO) ${ }^{2}$ project in Italy. Interferometers with baselines of several hundred meters are under construction in the British/German Cooperation for Gravity Wave Experiment (GEO) 600 (Ref. 3) projects in Germany and the Japanese Interferometric Gravitational-Wave Detector Project (TAMA). ${ }^{4}$ These kilometer-scale detectors will be sensitive to relative displacements of their test masses of the order of $10^{-19}$ to $10^{-20} \mathrm{~m} / \sqrt{\mathrm{Hz}}$ in the frequency band from approximately 10 to several $1000 \mathrm{~Hz}$. At frequencies above approximately $300 \mathrm{~Hz}$ the dominant noise source is expected to be photon shot noise.

The sensitivity limit imposed by photon shot noise depends on the optical configuration of the inter-

When this research was performed, the authors were with the Laser Interferometer Gravitational-Wave Observatory (LIGO), California Institute of Technology, Pasadena, California 91125. T. T. Lyons is now with the Mission Research Corporation, 3625 Del Amo Boulevard, Suite 215, Torrance, California 90503. M. W. Regehr is now with the Jet Propulsion Laboratory, Mail Stop 171113, 4800 Oak Grove Drive, Pasadena, California 91109-8099. F. J. Raab (raab_f@ligo.caltech.edu) is now with the LIGO Hanford Observatory, P.O. Box 1970, S9-02, Richland, Washington 99352.

Received 28 January 2000; revised manuscript received 12 September 2000.

0003-6935/00/366761-10\$15.00/0

(C) 2000 Optical Society of America ferometer as well as the technique employed to read out the relative positions of the test masses. There has been considerable effort devoted to exploring novel optical configurations and readout schemes that improve the shot-noise-limited performance of the detectors for a given laser power without requiring unreasonably high power levels in the interferometer. ${ }^{5-7}$

The optical configuration selected for the initial LIGO, VIRGO, and TAMA detectors is a powerrecycled interferometer with Fabry-Perot arm cavities $^{8,9}$ as shown in Fig. 1. A passing gravitational wave incident from directly overhead will produce a fluctuating strain that stretches one arm of the interferometer and contracts the other arm for half of the gravitational-wave period. The interferometer measures the change in the difference between the arm lengths in a way that is directly analogous to a Michelson interferometer. The Fabry-Perot cavities are held on resonance by length-control servos, and the beam splitter is controlled so that the light returning from the two arms interferes destructively at the antisymmetric port. This light interferes constructively at the symmetric port, with light returning toward the laser in accordance with energy conservation. The small deviation from resonance induced by a passing gravitational wave will cause the phase of the light reflected from the arms to change, spoiling the destructive interference at the antisymmetric port. This gives rise to the gravitational-wave signal. The recycling mirror improves the shot-noise-limited sensitivity by redirecting the light returning to the laser back into the interferometer. The recycling mirror must be positioned so that the light it reflects back into the inter- 


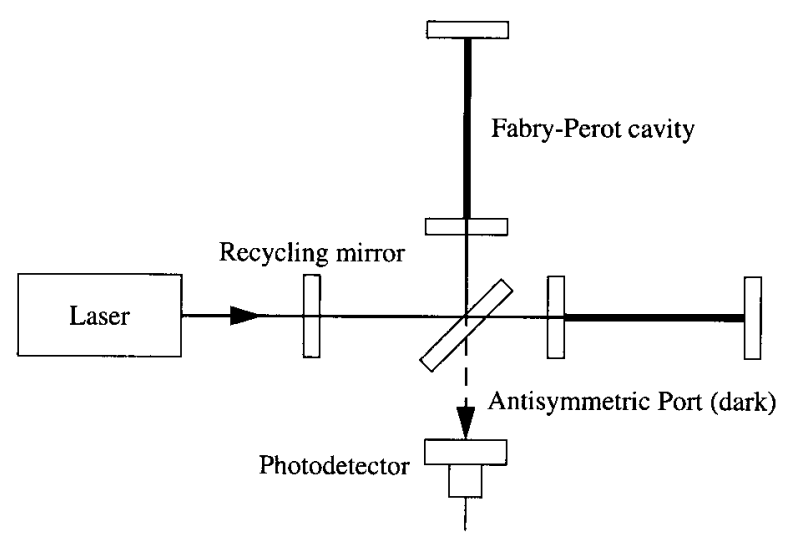

Fig. 1. Power-recycled interferometer with Fabry-Perot arm cavities.

ferometer interferes constructively with the light transmitted through it from the laser.

Calculating the shot-noise-limited sensitivity of a gravitational-wave interferometer is complicated by the fact that, to achieve adequate sensitivity, the light in the interferometer is phase modulated. The output light power is time varying at the phase modulation frequency and its harmonics. Thus the associated shot noise is nonstationary. Early treatments assumed that the shot noise was a white-noise source with a variance proportional to the time-averaged power incident on the photodetector. ${ }^{10-12}$ This approximation is useful to make order-of-magnitude predictions of shot-noise-limited sensitivity, but more accurate comparisons with experiment require one to include the effect of phase modulation and the demodulation waveform used. This has been done for a single Fabry-Perot cavity ${ }^{13,14}$ and for a delay-line interferometer, ${ }^{14}$ and the dependence of the shot noise on the demodulation waveform has been experimentally confirmed. ${ }^{15-17}$ A shot-noise-limited optical phase measurement has also been demonstrated at high optical power in a power-recycled Michelson interferometer. ${ }^{18}$

Fabry-Perot cavities are used in the arms of a power-recycled Michelson interferometer to provide a large amplification of the optical phase shift generated by a gravitational wave. Here we give a detailed derivation of the shot-noise-limited displacement sensitivity for such a power-recycled interferometer with Fabry-Perot arm cavities that includes the effect of phase modulation applied to the light incident on the interferometer. The resulting formula is compared directly with data from a 40-m-long, suspended-mirror, test interferometer incorporating optical recombination of light returning from the two arms. The empirical method we develop for this comparison accurately determines the shot-noise contribution to displacement noise even in the presence of other, larger noise contributions. The calculated shot-noise-limited displacement sensitivity and the experimental evaluation of

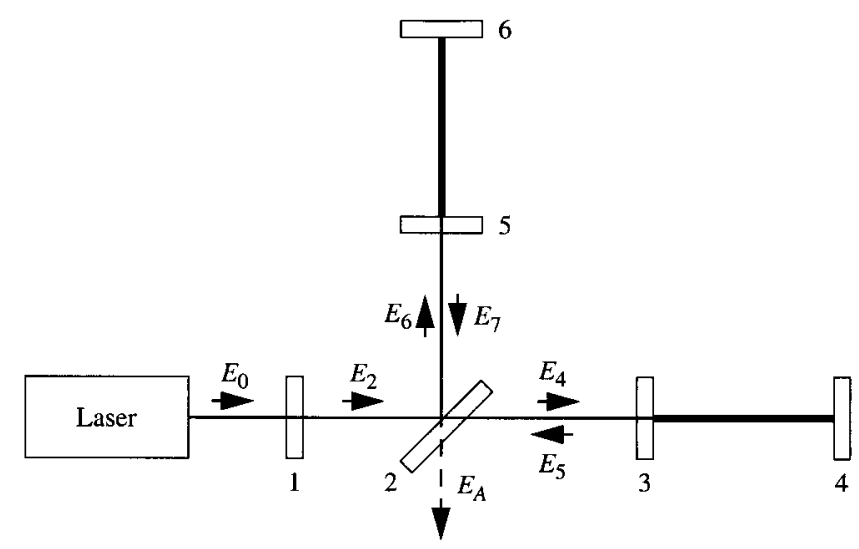

Fig. 2. Recycled interferometer with mirrors and optical fields labeled.

this noise contribution are in good agreement within experimental uncertainties.

In Section 2, we derive the response of the interferometer to mirror displacements. The power spectrum of shot noise at the demodulated signal output of the interferometer is given in Section 3. In Section 4 we describe an experimental confirmation of the calculated shot-noise contribution to a test interferometer that uses Fabry-Perot arms with a 40-m baseline.

\section{Interferometer Response}

A recycled interferometer with the mirrors and fields labeled is shown in Fig. 2. We specifically derive the contribution of shot noise in an interferometer that uses phase modulation on the incident light. ${ }^{19,20}$ However, the technique employed in this research is generally applicable to other configurations that apply phase modulation. The light incident from the laser is $E_{0}$. The light is phase modulated, with modulation depth $\Gamma$, between the laser and the interferometer. This impresses sidebands on the light at frequencies above and below the laser frequency (carrier), separated by the modulation frequency and its harmonics. The carrier light leaving the antisymmetric port is $E_{A}$, which is typically small in the absence of a signal because the antisymmetric port is held on a dark fringe for the carrier. Because of the asymmetry, the sidebands are not on a dark fringe at the antisymmetric port. We adopt the phase convention that the first-order sidebands have real amplitudes of opposite sign when incident on the beam splitter. The second-order sidebands have equal real amplitudes of the same sign. We neglect terms in the calculation of order $\Gamma^{3}$ because the modulation depth is assumed small. Thus we only need to consider up to second-order sidebands. The transmission of the $n$ th-order sideband from incidence on the beam splitter to the antisymmetric port is $\pm i \sin n \alpha$, where positive indicates the upper sideband and negative indicates the lower side- 


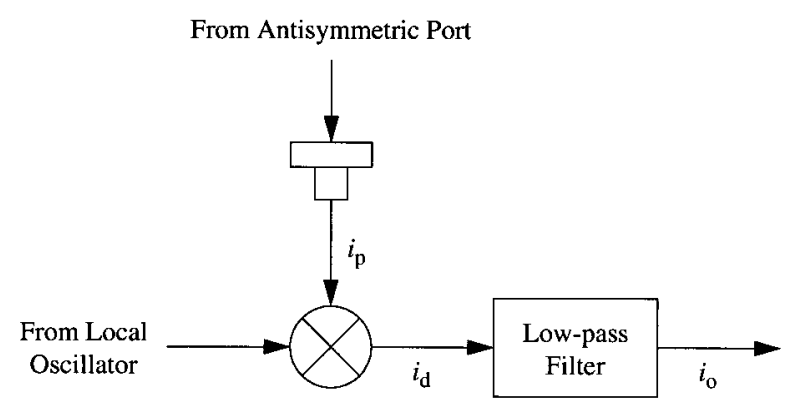

Fig. 3. Detection system for the antisymmetric port light.

band. ${ }^{21}$ The amplitude of the total complex field at the antisymmetric port is

$$
\begin{aligned}
E_{\text {anti }}= & E_{A}+i E_{+} \exp (i \omega t)+i E_{+} \exp (-i \omega t) \\
& +i E_{2_{+}} \exp (2 i \omega t)-i E_{2+} \exp (-2 i \omega t),
\end{aligned}
$$

where $\omega$ is the angular modulation frequency and $E_{+}$ and $E_{2+}$ are the magnitudes of the first-order and second-order sideband fields at the antisymmetric port.

The detection system is modeled as a photodetector, a demodulator, and a low-pass filter as shown in Fig. 3. The low-pass filter need not be explicitly built as a separate element following the mixer output. In practice, all the servo loops that derive their error signals from the mixer output have unity gain frequencies that are low compared with the modulation frequency. Thus in our analysis we ignore any signals at the mixer output that are at or above the modulation frequency.

A gravitational wave interacting with the detector will produce the same differential-mode signal as it does when we shake mirror 4 by some other means. If we displace mirror 4 such that $x_{4}=x_{0} \sin \Omega t$, for sufficiently small $x_{0}$ this will produce a signal at the antisymmetric port given by

$$
E_{\mathrm{A}}=E_{\mathrm{dc}}-i k E_{2} \frac{T_{3} r_{4}}{\left(1-r_{3} r_{4}\right)^{2}} \frac{x_{0} \sin (\Omega t+\psi)}{\left[1+\left(\Omega / \omega_{c}\right)^{2}\right]^{1 / 2}},
$$

where $k$ is the wave number of the light, $E_{\mathrm{dc}}$ is the field that is due to the contrast defect that comes from any noninterfering light on the photodetector, and $\psi$ is a phase factor irrelevant to this analysis. $\omega_{c}$ is the angular frequency of the so-called cavity pole,

$$
\omega_{c}=\frac{c}{2 l} \frac{1-r_{3} r_{4}}{r_{3} r_{4}},
$$

whose value is typically within the bandwidth of interest for gravitational waves. [Equation (2) is derived in greater detail in Appendix A.] The modulation sidebands do not resonate in the arm cavities and thus are not affected by the motion of mirror 4.

Expressing the fields in units of (photoelectrons/ s) $)^{1 / 2}$ simplifies the following formulas in our analysis. The photocurrent $i_{p}$ is

$$
\begin{aligned}
i_{p}= & \mid E_{A}+i E_{+} \exp (i \omega t)+i E_{+} \exp (-i \omega t) \\
& +i E_{2+} \exp (2 i \omega t)-\left.i E_{2+} \exp (-2 i \omega t)\right|^{2} \\
= & \left|E_{A}\right|^{2}+2 E_{+}{ }^{2}+4 E_{+} \operatorname{Im}\left(E_{A}\right) \cos \omega t \\
& +2 E_{+}{ }^{2} \cos 2 \omega t-4 E_{2+} \operatorname{Re}\left(E_{A}\right) \sin 2 \omega t .
\end{aligned}
$$

The photocurrent has components at zero frequency (dc), $\omega$, and $2 \omega$. The effect of the mixer and low-pass filter is to pick out the $\omega$ component, which is

$$
4 k E_{2} E_{+} \frac{T_{3} r_{4}}{\left(1-r_{3} r_{4}\right)^{2}} \frac{x_{0} \sin (\Omega t+\psi)}{\left[1+\left(\Omega / \omega_{c}\right)^{2}\right]^{1 / 2}} \cos \omega t .
$$

Because the photocurrent is modulated, it is important to treat the shot noise as a nonstationary random process and to consider the actual demodulation waveform used.

The effective demodulation waveform used in the 40-m interferometer is cosinusoidal. Square-wave demodulation is used at the mixer, but the bandpass filter, which is built into the photodiode and centered on the modulation frequency, makes this effectively cosinusoidal demodulation. This is because the square wave can be decomposed into a sum of cosine waves at odd multiples of the modulation frequency. Each cosine wave mixes with the corresponding component of the photocurrent to produce a signal after the low-pass filter. The bandpass filter on the photodiode effectively eliminates all these higherfrequency components in the photocurrent so that only the fundamental cosine wave demodulation term is important.

Multiplying the component of the photocurrent at $\omega$ by $\cos \omega t$, we obtain

$$
i_{d}=4 k E_{2} E_{+} \frac{T_{3} r_{4}}{\left(1-r_{3} r_{4}\right)^{2}} \frac{x_{0} \sin (\Omega t+\psi)}{\left[1+\left(\Omega / \omega_{c}\right)^{2}\right]^{1 / 2}} \cos ^{2} \omega t .
$$

The low-pass filter has a corner frequency that is much less than the modulation frequency. Thus the component of $\cos ^{2} \omega t$ near dc will pass through, but the component at $2 \omega$ will not, so that

$$
i_{o}=2 k E_{2} E_{+} \frac{T_{3} r_{4}}{\left(1-r_{3} r_{4}\right)^{2}} \frac{x_{0} \sin (\Omega t+\psi)}{\left[1+\left(\Omega / \omega_{c}\right)^{2}\right]^{1 / 2}} .
$$

We define $H(f)$ as the transfer function from $x_{0}$ to $i_{o}$ :

$$
\begin{aligned}
|H(f)| & \equiv\left|\frac{\tilde{\tau}_{o}(f)}{\tilde{x}_{0}(f)}\right| \\
& =2 k\left|E_{2}\right| E_{+} \frac{T_{3} r_{4}}{\left(1-r_{3} r_{4}\right)^{2}} \frac{1}{\left[1+\left(\Omega / \omega_{c}\right)^{2}\right]^{1 / 2}},
\end{aligned}
$$

where $\tilde{\imath}_{o}$ and $\tilde{x}_{0}$ denote the Fourier transforms of $i_{o}$ and $x_{0}$. 


\section{Noise}

To quantify the noise performance of the interferometer, we must characterize the random process $\mathbf{x}(t)$ corresponding to the output in the absence of any signal. We use boldfaced symbols in our notation here to mean random processes and $E\{\}$ to mean the expectation value or ensemble average. Early treatments of the shot noise assumed it was stationary and ignored the effect of the modulation of the photocurrent. Stationary noise is most conveniently represented by use of the one-sided power spectrum $S_{x x}(f)$ of $\mathbf{x}(t) . \quad S_{x x}(f)$ is defined as the Fourier transform of the autocorrelation function $R_{x x}(\tau)$ of $\mathbf{x}(t)$ :

$$
\begin{aligned}
R_{x x}(\tau) & =E\{\mathbf{x}(t+\tau) \mathbf{x}(t)\}, \\
S_{x x}(f) & =2 \int_{-\infty}^{\infty} R_{x x}(\tau) \exp (2 \pi i f \tau) \mathrm{d} \tau .
\end{aligned}
$$

If $\mathbf{x}(t)$ is the input of a linear system whose transfer function is $H(f)$ and $\mathbf{y}(t)$ is the output, then

$$
S_{y y}(f)=|H(f)|^{2} S_{x x}(f) .
$$

The output $\mathbf{i}_{\mathbf{o}}(t)$ of our model, in the absence of a signal, is not stationary because it fluctuates at the modulation frequency. However, it is cyclostationary, which is to say that for any $t$, the statistics of $\mathbf{i}_{\mathbf{o}}(t)$ are the same as those of $\mathbf{i}_{\mathbf{o}}(t+T)$, where $T$ is the period of modulation. In this situation, if we define the average autocorrelation and power spectrum

$$
\begin{aligned}
& \overline{R_{x x}}(\tau)=\frac{1}{T} \int_{t}^{t+T} R_{x x}\left(t^{\prime}+\tau, t^{\prime}\right) \mathrm{d} t^{\prime}, \\
& \overline{S_{x x}}(f)=2 \int_{-\infty}^{\infty} \overline{R_{x x}}(\tau) \exp (2 \pi i f \tau) \mathrm{d} \tau,
\end{aligned}
$$

then the relation

$$
\overline{S_{y y}}(f)=|H(f)|^{2} \overline{S_{x x}}(f)
$$

holds true. When we average in this way it is equivalent to modeling the time reference or phase of the cyclostationary process as a random variable that is uniformly distributed over one cycle. In this case the phase-randomized process is stationary. ${ }^{22,23}$

Our goal then is to calculate $\overline{S_{i_{i} i_{o}}}(f)$, the average power spectrum of the interferometer output. We begin by finding $\overline{S_{i_{d} i_{d}}}(f)$. The details of the derivation are in Appendix B; the result is

$$
\begin{aligned}
\overline{S_{i_{d} i_{d}}}(f)= & 3 E_{+}{ }^{2}+E_{\mathrm{dc}}{ }^{2}+\left(9 E_{+}{ }^{4}+6 E_{\mathrm{dc}}{ }^{2} E_{+}{ }^{2}\right. \\
& \left.+E_{\mathrm{dc}}{ }^{4}+4 E_{2+}{ }^{2} E_{\mathrm{dc}}{ }^{2}\right) \delta(2 \pi f-\omega) \\
& +\left(E_{+}{ }^{4}+4 E_{2+}{ }^{2} E_{\mathrm{dc}}{ }^{2}\right) \delta(2 \pi f-3 \omega) .
\end{aligned}
$$

This power spectrum has two sharp components, one at the modulation frequency and one at its third harmonic, as well as a broadband component. Only the broadband component interests us because it falls into the gravitational-wave frequency band. The
Table 1. Parameters for the 40-m Interferometer

\begin{tabular}{lcc}
\multicolumn{1}{c}{ Parameter } & Symbol & Value \\
\hline Mirror (power) transmissions & $T_{2}$ & 0.45 \\
& $T_{3}$ & $280 \mathrm{ppm}^{a}$ \\
& $T_{5}$ & $300 \mathrm{ppm}$ \\
Loss in each mirror & $T_{4}, T_{6}$ & $12 \mathrm{ppm}$ \\
& $L_{3}, L_{4}$ & $110 \mathrm{ppm}$ \\
Asymmetry & $L_{5}, L_{6}$ & $56 \mathrm{ppm}$ \\
Modulation frequency & $\delta$ & $50.8 \mathrm{~cm}$ \\
Modulation index & $f_{\bmod }$ & $12.33 \mathrm{MHz}$ \\
Contrast defect & $\Gamma$ & 1.49 \\
\hline
\end{tabular}

${ }^{a} \mathrm{ppm}$, parts per million.

low-pass filter in our model of the detection system will leave this part of the noise spectrum unaffected and will attenuate the higher-frequency components. Therefore

$$
\overline{S_{i_{o} i_{o}}}(f)=3 E_{+}{ }^{2}+E_{\mathrm{dc}}{ }^{2} \text {. }
$$

Finally, we obtain the displacement noise in one test mass equivalent to shot noise by substituting from Eqs. (8) and (14):

$$
\begin{aligned}
& \overline{S_{x_{4} x_{4}}}(f)^{1 / 2}=\frac{\overline{S_{i_{o} i_{o}}}(f)^{1 / 2}}{|H(f)|} \\
& =\frac{\left(3 E_{+}{ }^{2}+E_{\mathrm{dc}}{ }^{2}\right)^{1 / 2}}{2 k\left|E_{2}\right| E_{+}} \frac{\left(1-r_{3} r_{4}\right)^{2}}{T_{3} r_{4}}\left[1+\left(\frac{2 \pi f}{\omega_{c}}\right)^{2}\right]^{1 / 2} .
\end{aligned}
$$

\section{Experiment}

We derived the differential-mode displacement equivalent to shot noise in a power-recycled interferometer with Fabry-Perot arm cavities. The 40-m interferometer on the Caltech campus provides us with an opportunity to compare the theory with measurement. From April 1995 to August 1996 the 40- $\mathrm{m}$ interferometer was operated in a recombined configuration, which is identical to the planned initial LIGO and VIRGO configurations without the recycling mirror. ${ }^{24}$ A recombined interferometer can be treated as a power-recycled interferometer with a recycling mirror transmission equal to 1 .

To compare our theoretical expression for shot noise with laboratory measurements we must determine the reflectivities and transmissions of the arm cavity mirrors as well as the fields present in the interferometer. The transmissions and losses of the mirrors can be obtained from in situ measurements by use of the ringdown technique. ${ }^{25}$ This technique consists of building up a resonant field inside the cavity and then shutting off the power incident on the cavity. Observation of the time scale of the exponential decay of the light leaking out of the cavity allows a calculation of the mirror parameters. ${ }^{26}$ The measured parameters are shown in Table 1. 
Table 2. Parameters used in the Shot-Noise Calculation

\begin{tabular}{lc}
\hline Name & Value \\
\hline$V_{\max }$ & $1.1 \mathrm{~V}$ \\
$V_{\min }$ & $20 \mathrm{mV}$ \\
$R$ & $50 \Omega$ \\
$\Gamma$ & 0.705 \\
$M$ & 0.77 \\
$\alpha$ & 0.132 \\
$T_{3}$ & $280 \mathrm{ppm}$ \\
$r_{3}$ & 0.999805 \\
$r_{4}$ & 0.999938 \\
\hline
\end{tabular}

The fields in the interferometer, however, are not available for direct measurement. Instead, we measure the dc voltage by passing the antisymmetric port photocurrent through a known resistor. We record the minimum voltage when the interferometer is in lock $\left(V_{\min }\right)$, and the maximum voltage is observed when the arm cavities are out of lock and the beam splitter is allowed to swing freely $\left(V_{\max }\right)$. The modulation depth $\Gamma$ is measured with an optical spectrum analyzer. The fields are then found from

$$
\begin{aligned}
& E_{2}=\left(\frac{V_{\text {max }}}{R e}\right)^{1 / 2} J_{0}(\Gamma), \\
& E_{+}=\left(\frac{V_{\text {max }}}{R e}\right)^{1 / 2} J_{1}(\Gamma) \sin \alpha, \\
& E_{\text {dc }}=\left(\frac{V_{\text {min }}}{R e}-2{E_{+}}^{2}\right)^{1 / 2},
\end{aligned}
$$

where $R$ is the resistance in series with the photodiode and $e$ is the charge of the electron in Coulombs. (Note that for comparison with the experiment, we continue to write the fields in units of (photoelectrons $/ \mathrm{s})^{1 / 2}$ as we did for the theoretical expressions.)

To include the effect of light that is not mode matched properly into the arm cavities, we also measure the mode-matching fraction $M$ :

$$
M \approx \frac{1-R_{\text {arm }}}{1-R_{\text {theory }}},
$$

where $R_{\text {arm }}$ is the reflectivity of the arm cavities on resonance and $R_{\text {theory }}$ is the theoretical reflectivity for a perfectly aligned cavity with the same mirror transmissions and losses. The mode-matching fraction affects the shot-noise limit because only the light that could mode match into the cavities produces the signal. Mode matching does not affect the noise except as already accounted for in $E_{\mathrm{dc}}$. Thus the effective magnitude of $E_{2}$ and $E_{+}$in the denominator of the

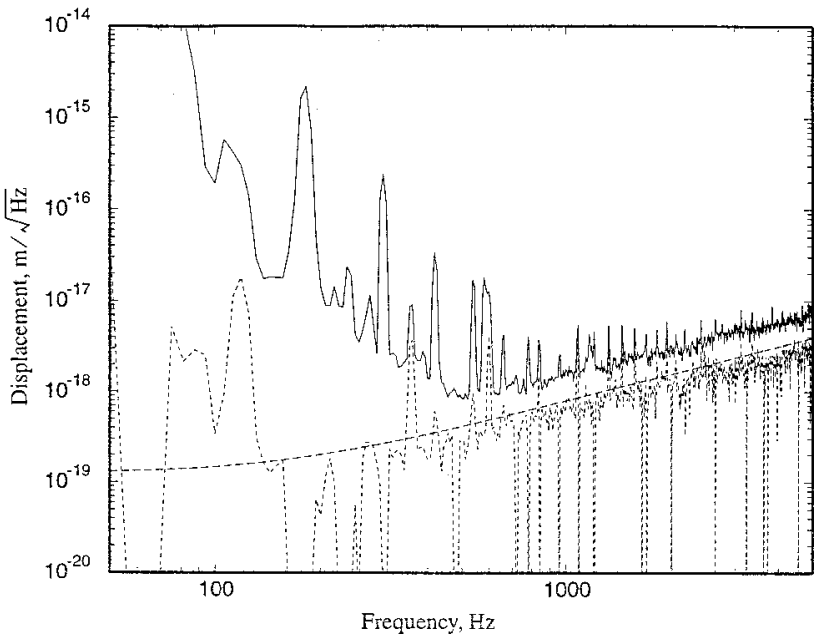

Fig. 4. Calculated shot-noise contribution to the interferometer displacement spectrum (long-dashed curve), with an empirical measurement of the shot-noise contribution (short-dashed curve) and interferometer displacement spectrum taken shortly before 10 January 1996 (solid curve).

shot-noise expression [Eqs. (15)] is reduced by $\sqrt{M}$. So,

$$
\overline{S_{\Delta}}(f)^{1 / 2}=\frac{\left(3 E_{+}{ }^{2}+E_{\mathrm{dc}}{ }^{2}\right)^{1 / 2}}{2 k M\left|E_{2}\right| E_{+}} \frac{\left(1-r_{3} r_{4}\right)^{2}}{T_{3} r_{4}}\left[1+\left(\frac{2 \pi f}{\omega_{c}}\right)^{2}\right]^{1 / 2} .
$$

The parameters used in the shot-noise calculation are shown in Table 2. The resulting curve is shown in Fig. 4.

We want to compare this calculated curve to with empirical measurement of the shot-noise contribution to the gravitational-wave signal (discussed below) and with the interferometer displacement spectrum taken at the time these measurements were performed. We can obtain the interferometer displacement spectrum by monitoring a test point in the servo system electronics that is used to control differences in the lengths of the Fabry-Perot cavities when the interferometer is held on resonance with a dark fringe at the antisymmetric port. This signal can then be calibrated when a mirror is actuated (mirror 4 of Fig. 2) to produce known sinusoidal displacements at a frequency that is swept through the frequency range of interest. We can obtain an empirical measurement of the shot-noise contribution to the gravitational-wave signal by blocking the laser light and shining incandescent light on the antisymmetric photodiode such that the photocurrent is the same as in normal operation. The gravitationalwave readout equivalent to this shot noise can then be calibrated, provided that the effect of the loop gain of servo systems controlling the interferometer is properly taken into account. With the interferometer in lock, the shot-noise signal is suppressed by the differential-mode loop gain. When the laser light is 


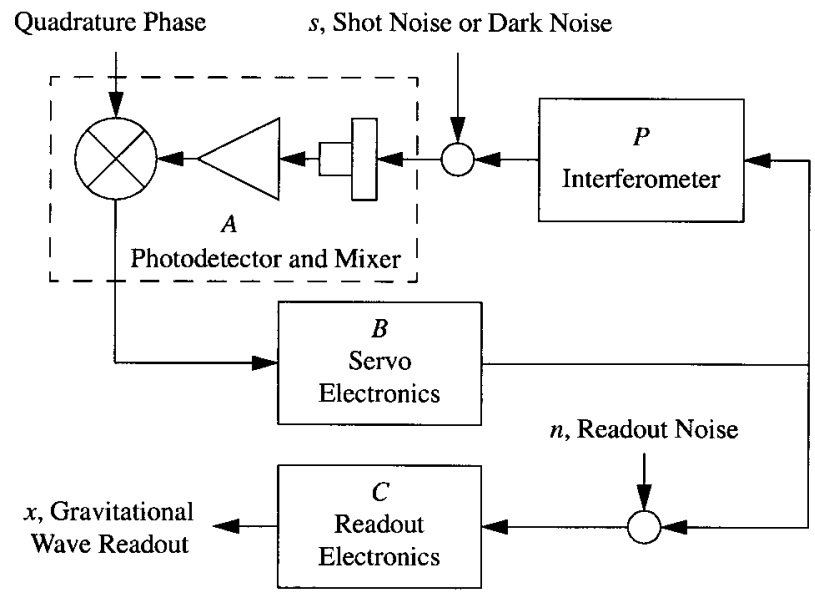

Fig. 5. Differential-mode servo loop with shot-noise, dark-noise, and readout noise inputs.

blocked, the differential-mode loop is open and this suppression factor is no longer present.

The action of changing the loop gain on various noise sources can be illustrated by a simple loop analysis. The differential-mode servo loop, with the places where shot noise, dark noise of the photodiode, and readout noise would sum in, is shown in Fig. 5. The transfer functions from the noise inputs to the gravitational-wave readout in the open loop case (when the laser light is blocked) are

$$
\left.\frac{x}{s}\right|_{\text {open loop }}=\left.A B C \quad \frac{x}{n}\right|_{\text {open loop }}=C .
$$

With the loop closed during normal interferometer operation,

$$
\left.\frac{x}{s}\right|_{\text {closed loop }}=\left.\frac{A B C}{1-L} \quad \frac{x}{n}\right|_{\text {closed loop }}=C,
$$

where the open loop gain is $L=A B P$. Thus with the loop closed, shot noise and the dark noise of the photodiode are suppressed by $1 /(1-L)$ relative to the open loop measurement whereas the readout noise is unaffected.

The complete measurement procedure for the empirical measurement of the shot-noise limit shown in Fig. 4 follows. The transfer functions of the differential-mode servo loop are measured to obtain the loop correction factor $1 /(1-L)$. After taking an interferometer displacement spectrum and the transfer function necessary for calibration, we block the laser light. As a check of the readout noise, the input to the readout electronics is terminated in $50 \Omega$, and the power spectrum of the gravitational-wave readout is recorded. After reconnecting the readout electronics, we record the power spectrum of the gravitational-wave readout with no light on the antisymmetric photodiode. This is the dark-noise spectrum and should be well above the level that is due to noise in the readout electronics, as it was in every case. Finally, the photodiode was illuminated with incandescent light to achieve the same photocurrent as is present during normal interferometer operation. The resulting power spectrum is the shot-noise plus dark-noise spectrum. The power spectrum of shot noise alone is recovered by quadrature subtraction of the dark noise. The shot-noise power spectrum is then increased by $1 \mathrm{~dB}$ to reflect the fact that the measured fluctuations in the photocurrent from the photodiode are observed to be $1 \mathrm{~dB}$ greater for the green laser light than for incandescent light producing the same dc photocurrent. (The origin of this effect is not understood. ${ }^{27}$ ) This spectrum is then divided by $1 /(1-L)$, to account for the differential-mode loop gain, and calibrated as usual to convert it into an equivalent amount of displacement noise.

The resulting empirical measurement of the shotnoise-equivalent displacement is shown as the shortdashed curve in Fig. 4. Ideally, the shot-noise power spectrum should have been larger than the darknoise spectrum by a reasonable margin. In fact, for the measurement shown in Fig. 4, there was only approximately a $3-\mathrm{dB}$ margin which is why the resulting estimate for the shot-noise contribution alone appears noisy.

It is evident from Fig. 4 that the measured contribution of shot noise to the interferometer output is less than the total noise. A significant amount of effort was made to understand the observed excess noise in the interferometer displacement spectrum. It is suggestive that the shape of the spectrum matches that predicted for shot noise above approximately $600 \mathrm{~Hz}$. This would be the case for any noise source that is equivalent to white noise at the demodulator output. We explicitly tested for a number of potential noise sources, including intensity noise, frequency noise, beam-splitter motion, and shot noise in the auxiliary signals. None of these noise sources were found to limit the interferometer displacement spectrum above $600 \mathrm{~Hz}$.

Intensity noise can contribute to the displacement spectrum because of in-band $(f<10-\mathrm{kHz})$ fluctuations as well as fluctuations at frequencies near the radio-frequency (rf) modulation frequency. We estimated the in-band contribution by injecting white intensity noise at a level to clearly show up in the interferometer output above the observed noise level. This drive level was then doubled to check for linearity, which did produce a 6 - $\mathrm{dB}$ increase in the interferometer noise level. By comparing the increase in the interferometer displacement spectrum with the increase in the intensity noise spectrum, we could set a limit on the intensity noise contribution. The inband intensity noise contribution was $3 \times 10^{-19}$ $\mathrm{m} / \sqrt{\mathrm{Hz}}$ at $850 \mathrm{~Hz}$ and was relatively flat from 500 to $1000 \mathrm{~Hz}$. By contrast, the interferometer noise was $(1-2) \times 10^{-18} \mathrm{~m} / \mathrm{V} \mathrm{Hz}$ over this frequency range. A test for rf intensity noise is to misalign all the test masses except for a single vertex mass so that light incident on the interferometer is reflected back to the photodiodes. After we measure the demodulated signal at the symmetric or antisymmetric photo- 
diodes, the laser light is blocked, and the same amount of power is applied to the photodiode with an incandescent light source. Above $200 \mathrm{~Hz}$ the spectra of the resulting demodulated signal were identical in both cases. This confirms that the intensity noise of the light is shot-noise limited near the rf modulation frequency.

We estimated the frequency noise contribution to the interferometer output by injecting a monochromatic frequency deviation and observing the resulting peaks in the frequency-control servo signal and in the interferometer output. We expect the frequency noise feeding through to the interferometer output to be essentially constant over some small region around the injected peak. By comparing the peakto-background measurements, we determined the estimated frequency noise contribution to the interferometer output at $750 \mathrm{~Hz}$ to be less than $7 \times$ $10^{-20} \mathrm{~m} / \sqrt{\mathrm{Hz}}$.

We estimated the contribution from in-band fluctuations in the beam-splitter position by measuring the transfer function between the beam-splitter feedback signal and the interferometer output. The ambient spectrum of the beam-splitter feedback was multiplied by this transfer function to find the estimate. Above $600 \mathrm{~Hz}$, the contribution to the interferometer output from beam-splitter motion is more than $40 \mathrm{~dB}$ below the observed spectrum.

Shot noise in the auxiliary servo signals may feed through onto the gravitational readout signal. The error signals for these servos are measured at the symmetric photodiode. We placed an attenuator before the symmetric photodiode to halve the laser light and then used an incandescent light source to increase the power on the photodiode by a factor of 4 . We saw no observable change in the gravitationalwave spectrum.

\section{Conclusion}

We have given a derivation of the shot-noise-limited sensitivity of a power-recycled interferometer with Fabry-Perot arm cavities. The result was compared with data from the $40-\mathrm{m}$ interferometer operated in a recombined configuration without a recycling mirror. In particular an empirical measurement of the contribution of shot noise to the interferometer was possible, even in the presence of other noise sources. This empirical measurement of the shot-noise contribution agrees with the calculation to within the uncertainties of the parameters in the calculation and in the calibration, typically a few decibels.

We determined that the interferometer was not limited by shot noise at any frequency. Over the frequency range from 500 to $1500 \mathrm{~Hz}$, the interferometer exhibited a noise-equivalent displacement that was typically $(1-2) \times 10^{-18} \mathrm{~m} / \sqrt{\mathrm{Hz}}$ (except for narrow features associated with mechanical resonances and line harmonics), increasing to approximately $7 \times 10^{-18} \mathrm{~m} / \sqrt{\mathrm{Hz}}$ at $5000 \mathrm{~Hz}$. The measured contribution of shot noise to the noiseequivalent displacement varied from $3 \times 10^{-19}$ $\mathrm{m} / \sqrt{\mathrm{Hz}}$ to approximately $3 \times 10^{-18} \mathrm{~m} / \sqrt{\mathrm{Hz}}$ over the frequency range $500-5000 \mathrm{~Hz}$. The calculated shotnoise-equivalent displacement, by use of the measured parameters in Table 2, was larger than the measured displacement by approximately $3 \mathrm{~dB}$. This is comparable with our estimate of measurement uncertainties.

We confirmed that shot noise was not the dominant noise by attenuating the light leaving the antisymmetric port by $37.5 \%$ and directing light from an incandescent bulb onto the photodiode to raise the incident power by a factor of 3.2. We would expect a 7-dB increase in the interferometer displacement spectrum if it were limited by shot noise, but the largest increase seen anywhere in this frequency band was $4 \mathrm{~dB}$. Although the interferometer noise is not fully understood, it is clearly not shot-noise limited. A number of noise sources were explored and eliminated as significant noise contributions, including laser intensity and frequency fluctuations, beam-splitter motion, and shot noise on the auxiliary control signals derived from the symmetric port. A leading candidate to explain the excess noise is scattered light, most likely in the vertex area. There was significant scattering from optics situated inside the beam-splitter's vacuum chamber, and we were not able to extensively test whether this caused the noise excess. However, the presence of this noise did not degrade our ability to confirm the shot-noise contribution to the observed displacement spectrum as shown in Fig. 4.

The methods used here for calculation of the shot-noise contribution and for the empirical measurement of this contribution are quite general. They are directly applicable to the large-scale gravitational-wave detectors currently under construction for LIGO and VIRGO, and they can be readily adapted for other interferometer configurations.

\section{Appendix A: Effect of Shaking an End Mirror}

Here we derive the effect on $E_{A}$ of shaking mirror 4 at frequency $\Omega$ as mentioned in Section 2. To do this we need to calculate the field reflected from the arm cavity $E_{5}$ in terms of the incident field $E_{4}$. This is done in two steps. First we solve for $E_{5}$ given a small dc displacement of mirror 4 . Then we generalize this result to frequencies in the gravitationalwave band.

Consider a small displacement of mirror 4 away from the carrier resonance. Let $x_{4}=0$ on resonance so that $x_{4}=x_{0}$ after the displacement. We define the arm cavity reflectivity away from resonance to be $r_{\text {arm }}(\phi)$ such that $E_{5}=r_{\text {arm }}(\phi) E_{4}$, where

$$
\begin{aligned}
r_{\mathrm{arm}}(\phi) & =\frac{r_{3}-\left(1-L_{3}\right) r_{4} \exp (i \phi)}{1-r_{3} r_{4} \exp (i \phi)}, \\
\phi & =2 k x_{4} .
\end{aligned}
$$


Here $L_{3}$ is the loss associated with mirror 3 (assumed equal to the loss from mirror 4). When we Taylor expand $r_{\text {arm }}(\phi)$, then

$$
E_{5}=E_{4}\left(r_{\text {arm }}\left|x_{4}=0+\frac{\mathrm{d} r_{\text {arm }}}{\mathrm{d} x_{4}}\right|_{x_{4}=0} x_{0}+\ldots\right) .
$$

Taking the derivative and noting $\mathrm{d} \phi / \mathrm{d} x_{4}=2 k$, for sufficiently small $x_{0}$ we can write

$$
E_{5}=E_{4}\left[\frac{r_{3}-\left(1-L_{3}\right) r_{4}}{1-r_{3} r_{4}}-2 i k \frac{T_{3} r_{4}}{\left(1-r_{3} r_{4}\right)^{2}} x_{0}\right] \text {. }
$$

Now let $x_{4}=x_{0} \sin \Omega t$. Note that in the small amplitude limit considered here, when we shake the rear mirror at frequency $\Omega$, the light reflected from the mirror is phase modulated. This impresses sidebands on the reflected light at frequencies $\Omega$ above and below the carrier frequency. The transmission of these sidebands from the rear mirror through the cavity is

$$
t_{\mathrm{arm}}(\phi)=\frac{t_{3} \exp (i \phi / 2)}{1-r_{3} r_{4} \exp (i \phi)} .
$$

Now $\phi=2 \omega l / c$ where $\omega$ is the angular frequency of the light and $l$ is the length of the cavity. The frequency of the light with the impressed sidebands from the mirror motion is $\omega=\omega_{0} \pm \Omega$ where $\omega_{0}$ is the carrier resonance frequency. We assume $\Omega$ is small compared to the cavity free spectral range. (The arm cavities for LIGO will have a free spectral range of $37.5 \mathrm{kHz}$, and the free spectral range for the $40-\mathrm{m}$ interferometer was $3.75 \mathrm{MHz}$.) We can approximate

$$
\begin{aligned}
\exp (i \phi) & =\exp \left[2 i\left(\omega_{0} \pm \Omega\right) \frac{l}{c}\right] \\
& =\exp \left(i \Omega \frac{2 l}{c}\right) \approx 1 \pm i \Omega \frac{2 l}{c} \\
t_{\mathrm{arm}}(\phi) & =\frac{t_{3}\left(1 \pm i \Omega \frac{l}{c}\right)}{1-r_{3} r_{4}\left(1 \pm i \Omega \frac{2 l}{c}\right)}
\end{aligned}
$$

This has a zero at angular frequency $c / l$, which is twice the cavity free spectral range. This zero is well above the gravitational-wave band and therefore not of interest. There is also a pole at angular frequency

$$
\omega_{c}=\frac{c}{2 l} \frac{1-r_{3} r_{4}}{r_{3} r_{4}}
$$

This is the so-called cavity pole. It will typically be important and lie in the gravitational-wave band.

We can now generalize from the dc case by noting that all the frequency dependence in the transfer function from $x_{4}$ to $E_{5}$ is contained in a single pole:

$$
\begin{aligned}
E_{5}= & E_{4}\left\{\frac{r_{3}-\left(1-L_{3}\right) r_{4}}{1-r_{3} r_{4}}\right. \\
& \left.-2 i k \frac{T_{3} r_{4}}{\left(1-r_{3} r_{4}\right)^{2}} \frac{x_{0} \sin (\Omega t+\psi)}{\left[1+\left(\Omega / \omega_{c}\right)^{2}\right]^{1 / 2}}\right\},
\end{aligned}
$$

where $\psi$ is a phase factor that is irrelevant for this analysis.

If we assume negligible losses and equal power transmission and reflection in the beam splitter, then

$$
E_{4}=\frac{E_{2}}{\sqrt{2}}, \quad E_{6}=-\frac{E_{2}}{\sqrt{2}} .
$$

The field at the antisymmetric port is

$$
E_{A}=\frac{1}{\sqrt{2}} E_{5}+\frac{1}{\sqrt{2}} E_{7} .
$$

Now

$$
\begin{aligned}
& E_{7}=E_{6} \frac{r_{5}-\left(1-L_{5}\right) r_{6}}{1-r_{5} r_{6}}, \\
& E_{A}=E_{\mathrm{dc}}-i k E_{2} \frac{T_{3} r_{4}}{\left(1-r_{3} r_{4}\right)^{2}} \frac{x_{0} \sin (\Omega t+\psi)}{\left[1+\left(\Omega / \omega_{c}\right)^{2}\right]^{1 / 2}},
\end{aligned}
$$

where $E_{\mathrm{dc}}$ is the excess light at the antisymmetric port that is due to the imperfect matching of mirror parameters between the two arms or, more generally, any noninterfering sources of light on the photodetector.

\section{Appendix B: Average Power Spectrum of the Demodulator Output}

Here we derive the average power spectrum of the demodulator output $\overline{S_{i_{d} i_{d}}}(f)$ from the time-averaged autocorrelation function $R_{i_{d} i_{d}}(\tau)$ using the methods discussed in Ref. 23. To calculate $\overline{R_{i_{d} i_{d}}}(\tau)$, we first find the expectation value of the photocurrent $E\left\{\mathbf{i}_{\mathbf{p}}(t)\right\}$ in the absence of any signal. In this case $E_{A}=E_{\mathrm{dc}}$, thus from Eqs. (4),

$$
\begin{aligned}
E\left\{\mathbf{i}_{\mathbf{p}}(t)\right\}= & \left|E_{\mathrm{dc}}\right|^{2}+2 E_{+}{ }^{2}+4 E_{+} \operatorname{Im}\left(E_{\mathrm{dc}}\right) \cos \omega t \\
& +2 E_{+}{ }^{2} \cos 2 \omega t-4 E_{2+} \operatorname{Re}\left(E_{\mathrm{dc}}\right) \sin 2 \omega t \\
= & {E_{\mathrm{dc}}}^{2}+2 E_{+}{ }^{2}+2 E_{+}{ }^{2} \cos 2 \omega t \\
& -4 E_{2+} E_{\mathrm{dc}} \sin 2 \omega t .
\end{aligned}
$$

$E_{\mathrm{dc}}$ has no imaginary part, because if it had then the length-control servo would induce a differential change in the cavity lengths to cancel it.

The total number of electrons having left the photodetector since some initial time $t=0$ is modeled as a nonuniform Poisson process. A Poisson process $\mathbf{q}(t)$ is a random process that is constant except for unit increments at random points in time $\mathbf{t}_{\mathbf{i}}$. We label $\lambda(t)$ the density of the points of $\mathbf{t}_{\mathbf{i}}$. The term nonuniform applies if the density of points is a func- 
tion of time. We identify $\lambda(t)=E\left\{\mathbf{i}_{\mathbf{p}}(t)\right\}$. We write this as

$$
\lambda(t)=a+b \cos 2 \omega t+c \sin 2 \omega t .
$$

The photodetector output current is then a random process that is the derivative of a Poisson process. This is called a process of Poisson impulses.

$$
\begin{aligned}
\mathbf{i}_{\mathbf{p}}(t) & =\frac{\mathrm{d} \mathbf{q}(t)}{\mathrm{d} t} \\
& =\sum_{i} \delta\left(t-\mathbf{t}_{\mathbf{i}}\right) .
\end{aligned}
$$

The autocorrelation of a nonuniform Poisson process is ${ }^{28}$

$$
R_{q q}\left(t_{1}, t_{2}\right)=\left\{\begin{array}{ll}
\int_{0}^{t_{2}} \lambda(t) \mathrm{d} t\left[1+\int_{0}^{t_{1}} \lambda(t) \mathrm{d} t\right] & t_{1}>t_{2} \\
\int_{0}^{t_{1}} \lambda(t) \mathrm{d} t\left[1+\int_{0}^{t_{2}} \lambda(t) \mathrm{d} t\right] & t_{2}>t_{1}
\end{array} .\right.
$$

The autocorrelation of the derivative of a random process is given by ${ }^{29}$

$$
R_{q^{\prime} q^{\prime}}\left(t_{1}, t_{2}\right)=\frac{\partial^{2} R_{x x}\left(t_{1}, t_{2}\right)}{\partial t_{1} \partial t_{2}} .
$$

Because $\mathbf{i}_{\mathbf{p}}(t)=x^{\prime}(t)$ we only have to substitute into Eq. (B5) to find the autocorrelation for the photocurrent. So

$$
\begin{aligned}
R_{i_{p} i_{p}}\left(t_{1}, t_{2}\right) & =\frac{\partial^{2} R_{q q}\left(t_{1}, t_{2}\right)}{\partial t_{1} \partial t_{2}} \\
& = \begin{cases}\lambda\left(t_{1}\right) \lambda\left(t_{2}\right) & t_{1}>t_{2} \\
\lambda\left(t_{2}\right) \lambda\left(t_{1}\right) & t_{2}>t_{1}\end{cases}
\end{aligned}
$$

However, there is a discontinuity in the derivative at $t_{1}=t_{2}$. Thus

$$
R_{i_{p} i_{p}}\left(t_{1}, t_{2}\right)=\lambda\left(t_{1}\right) \lambda\left(t_{2}\right)+\lambda\left(t_{1}\right) \delta\left(t_{1}-t_{2}\right) .
$$

We can use this result to find the time-averaged autocorrelation of the demodulator output $\mathbf{i}_{\mathbf{d}}(t)=$ $\mathbf{i}_{\mathbf{p}}(t) \cos \omega t$ :

$$
\begin{aligned}
R_{i_{d} i_{d}}(t+\tau, t)= & E\left\{\mathbf{i}_{\mathbf{p}}(t+\tau) \cos \omega(t+\tau) \mathbf{i}_{\mathbf{p}}(t) \cos \omega t\right\} \\
= & E\left\{\mathbf{i}_{\mathbf{p}}(t+\tau) \mathbf{i}_{\mathbf{p}}(t)\right\} \cos \omega(t+\tau) \cos \omega t \\
= & {[\lambda(t+\tau) \lambda(t)+\lambda(t+\tau) \delta(\tau)] } \\
& \times \cos \omega(t+\tau) \cos \omega t, \\
\overline{R_{i_{d} i_{d}}}(\tau)= & \frac{1}{T} \int_{0}^{T} R_{i_{d} i_{d}}(t+\tau, t) \mathrm{d} t \\
= & \frac{1}{T} \int_{0}^{T}[\lambda(t+\tau) \lambda(t)+\lambda(t+\tau) \delta(\tau)] \\
& \times \cos \omega(t+\tau) \cos \omega t \mathrm{~d} t,
\end{aligned}
$$

where $T$ is the modulation period.
To find the average power spectrum, we take the Fourier transform of the average autocorrelation:

$$
\overline{S_{i_{d} i_{d}}}(f)=2 \int_{-\infty}^{\infty} \overline{R_{i_{d} i_{d}}}(\tau) \exp (2 \pi i f \tau) \mathrm{d} \tau .
$$

We evaluate the two terms in Eqs. (B9) one at a time. The first term yields

$$
\begin{aligned}
\frac{1}{T} \int_{0}^{T} \lambda(t+\tau) \lambda(t) \cos \omega(t+\tau) \cos \omega t \mathrm{~d} t \\
=\frac{1}{T} \int_{0}^{T}(a+b \cos 2 \omega t+c \sin 2 \omega t) \\
\quad \times[a+b \cos 2 \omega(t+\tau)+c \sin 2 \omega(t+\tau)] \\
\quad \times \cos \omega(t+\tau) \cos \omega t \mathrm{~d} t \\
=\frac{1}{2}\left\{\left[a^{2}+a b+\frac{1}{4}\left(b^{2}+c^{2}\right)\right] \cos \omega \tau\right. \\
\left.\quad+\frac{1}{4}\left(b^{2}+c^{2}\right) \cos 3 \omega \tau\right\} .
\end{aligned}
$$

Substituting Eq. (B11) into Eq. (B10) yields

$2 \int_{-\infty}^{\infty} \frac{1}{2}\left\{\left[a^{2}+2 a b+\frac{1}{4}\left(b^{2}+c^{2}\right)\right] \cos \omega \tau\right.$

$$
\begin{array}{r}
\left.+\frac{1}{4}\left(b^{2}+c^{2}\right) \cos 3 \omega \tau\right\} \exp (2 \pi i f \tau) \mathrm{d} \tau \\
=\left[a^{2}+a b+\frac{1}{4}\left(b^{2}+c^{2}\right)\right] \delta(2 \pi f-\omega) \\
+\frac{1}{4}\left(b^{2}+c^{2}\right) \delta(2 \pi f-3 \omega) .
\end{array}
$$

To evaluate the second term we reverse the order of integration:

$$
\begin{aligned}
& \frac{2}{T} \int_{-\infty}^{\infty} \int_{0}^{T} \lambda(t+\tau) \delta(\tau) \cos \omega(t+\tau) \cos \omega t \mathrm{~d} t \exp (2 \pi i f \tau) \mathrm{d} \tau \\
&=\frac{2}{T} \int_{0}^{T} \int_{-\infty}^{\infty} \lambda(t+\tau) \delta(\tau) \cos \omega(t+\tau) \\
& \quad \times \cos \omega t \exp (2 \pi i f \tau) \mathrm{d} \tau \mathrm{d} t \\
&=\frac{2}{T} \int_{0}^{T} \lambda(t) \cos ^{2} \omega t \mathrm{~d} t \\
&=\frac{2}{T} \int_{0}^{T}(a+b \cos 2 \omega t+c \sin 2 \omega t) \cos ^{2} \omega t \mathrm{~d} t \\
&= a+\frac{b}{2} .
\end{aligned}
$$


Therefore the average power spectrum of the demodulator output is

$$
\begin{aligned}
& \overline{S_{i_{d} i_{d}}}(f)=3 E_{+}{ }^{2}+E_{\mathrm{dc}}{ }^{2}+\left(9 E_{+}{ }^{4}+6 E_{\mathrm{dc}}{ }^{2} E_{+}{ }^{2}\right. \\
& \left.+E_{\mathrm{dc}}{ }^{4}+4 E_{2+}{ }^{2} E_{\mathrm{dc}}{ }^{2}\right) \delta(2 \pi f-\omega) \\
& +\left(E_{+}{ }^{4}+4 E_{2+}{ }^{2} E_{\mathrm{dc}}{ }^{2}\right) \delta(2 \pi f-3 \omega) \text {. }
\end{aligned}
$$

We thank the members of the LIGO project for their encouragement and helpful discussions. We particularly thank S. E. Whitcomb, R. E. Spero, and R. Flaminio for helpful advice and insights. This research is supported by the National Science Foundation under cooperative agreement PHY9210038.

\section{References and Notes}

1. A. Abramovici, W. E. Althouse, R. W. P. Drever, Y. Gursel, S. Kawamura, F. J. Raab, D. Shoemaker, L. Sievers, R. E. Spero, K. S. Thorne, R. E. Vogt, R. Weiss, S. E. Whitcomb, and M. E. Zucker, "LIGO-the laser interferometer gravitational-wave observatory," Science 256, 325-333 (1992).

2. C. Bradaschia, R. Del Fabbro, A. Di Virgilio, A. Giazotto, H. Kautzky, V. Montelatici, D. Passuello, A. Brillet, O. Cregut, P. Hello, C. N. Man, P. T. Manh, A. Marraud, D. Shoemaker, J. Y. Vinet, F. Barone, L. Di Fiore, L. Milano, G. Russo, J. M. Aguirregabiria, H. Bel, J. P. Duruisseau, G. Ledenmat, P. Tourrenc, M. Capozzi, M. Longo, M. Lops, I. Pinto, G. Rotoli, T. Damour, S. Bonazzola, J. A. Marck, Y. Gourghoulon, L. E. Holloway, F. Fuligni, V. Iafolla, and G. Natale, "The VIRGO project: a wide band antenna for gravitational wave detection," Nucl. Instrum. Methods Phys. Res. A 289, 518-525 (1990).

3. K. Danzmann, H. Luck, A. Rudiger, R. Schilling, M. Schrempel, W. Winkler, J. Hough, G. P. Newton, N. A. Robertson, H. Ward, A. M. Campbell, J. E. Logan, D. I. Robertson, K. A. Strain, J. R. J. Bennett, V. Kose, M. Kuhne, B. F. Schutz, D. Nicholson, J. Shuttleworth, H. Welling, P. Aufmuth, R. Rinkleff, A. Tunnermann, and B. Willke, "GEO 600. A $600 \mathrm{~m}$ laser interferometric gravitational wave antenna," in First Edoardo Amaldi Conference on Gravitational Wave Experiments, E. Coccia, G. Pizzella, and F. Ronga, eds. (World Scientific, Singapore, 1995), pp. 100-111.

4. K. Tsubono, "300-m laser interferometer gravitational wave detector (TAMA300) in Japan," in First Edoardo Amaldi Conference on Gravitational Wave Experiments, E. Coccia, G. Pizzella, and F. Ronga, eds. (World Scientific, Singapore, 1995), pp. $112-114$.

5. B. J. Meers, "Recycling in laser-interferometric gravitationalwave detectors," Phys. Rev. D 38, 2317-2326 (1988).

6. J. Mizuno, K. A. Strain, P. Nelson, J. Chen, R. Schilling, A. Rüdiger, W. Winkler, and K. Danzmann, "Resonant sideband extraction: a new configuration for interferometric gravitational wave detectors," Phys. Lett. A 175, 273-276 (1993).

7. K. X. Sun, M. M. Feyer, E. Gustafson, and R. L. Byer, "Sagnac interferometer for gravitational-wave detection," Phys. Rev. Lett. 76, 3053-3056 (1996).

8. R. W. P. Drever, G. M. Ford, J. Hough, I. M. Kerr, A. J. Munley, J. R. Pugh, N. A. Robertson, and H. Ward, "A gravity-wave detector using optical cavity sensing," in Ninth International Conference on General Relativity and Gravitation, E. Schmutzer, ed. (Cambridge U. Press, Cambridge, UK, 1983).

9. R. W. P. Drever, "Interferometric detectors for gravitational radiation," in Gravitational Radiation, N. Deruelle and T. Piran, eds. (North-Holland, Amsterdam, 1983), pp. 321-328.

10. K. S. Thorne, "Gravitational radiation," in 300 Years of Gravitation, S. W. Hawking and W. Israel, eds. (Cambridge U. Press, Cambridge, UK, 1987), Eq. 115, p. 424.

11. J. Y. Vinet, B. Meers, C. N. Man, and A. Brillet, "Optimization of long-baseline optical interferometers for gravitational-wave detection," Phys. Rev. D 38, 433-447 (1988).

12. D. Shoemaker, P. Fritschel, J. Giaime, N. Christensen, and R. Weiss, "Prototype Michelson interferometer with Fabry-Perot cavities," Appl. Opt. 30, 3133-3138 (1991).

13. S. Whitcomb and R. Spero, "Shot noise in the Caltech $40 \mathrm{~m}$ interferometer," LIGO internal document, LIGO-T850002-00-D (California Institute of Technology, Pasadena, Calif., 1985).

14. T. M. Niebauer, R. Schilling, K. Danzmann, A. Rüdiger, and W. Winkler, "Nonstationary shot noise and its effect on the sensitivity of interferometers," Phys. Rev. A 43, 5022-5029 (1991).

15. B. J. Meers and K. A. Strain, "Modulation, signal, and quantum noise in interferometers," Phys. Rev. A 44, 4693-4703 (1991).

16. N. Mio and K. Tsubono, "Observation of an effect due to nonstationary shot noise," Phys. Lett. A 164, 255-258 (1992).

17. M. B. Gray, A. J. Stevenson, H. A. Bachor, and D. E. McClelland, "Harmonic demodulation of nonstationary shot noise," Opt. Lett. 18, 759-761 (1993).

18. P. Fritschel, G. Gonzalez, B. Lantz, P. Saha, and M. Zucker, "High power interferometric measurement limited by quantum noise and application to detection of gravitational waves," Phys. Rev. Lett. 80, 3181-3184 (1998).

19. M. W. Regehr, F. J. Raab, and S. E. Whitcomb, "Demonstration of a power-recycled Michelson interferometer with Fabry-Perot arms by frontal modulation," Opt. Lett. 20, 1507-1509 (1995).

20. R. Flaminio and H. Heitmann, "Longitudinal control of an interferometer for the detection of gravitational waves," Phys. Lett. A 214, 112-122 (1996).

21 . The $n$ th-order sideband transmission to the antisymmetric port is $t_{n \pm}=1 / 2\left\{\exp \left[2 i(k \pm n K) l_{1}\right]-\exp \left[2 i(k \pm n K) l_{2}\right]\right\}$ where $K$ equals the wave number at the modulation frequency. Let $l=1 / 2\left(l_{1}+l_{2}\right)$. Then, neglecting unimportant phase factors and accounting for the carrier being on a dark fringe yield

$$
\begin{aligned}
t_{n \pm}= & 1 / 2\{\exp 2 i[(k \pm n K)(l+\delta / 2)] \\
& -\exp [2 i(k \pm n K)(l-\delta / 2)]\} \\
= & 1 / 2[\exp ( \pm i n K \delta)-\exp (\mp i n K \delta)] \\
= & \pm i \sin n \alpha .
\end{aligned}
$$

22. W. A. Gardner and L. E. Franks, "Characterization of cyclostationary random signal processes," IEEE Trans. Inf. Theory IT-21, 4-14 (1975).

23. A. Papoulis, Probability, Random Variables, and Stochastic Processes, 3rd ed. (McGraw-Hill, San Francisco, Calif., 1991), pp. 373-374.

24. T. Lyons, A. Kuhnert, F. J. Raab, J. E. Logan, D. Durance, R. E. Spero, S. Whitcomb, and B. Kells, "Optical recombination of the 40-m interferometer," LIGO internal document LIGOT000095-00-D (California Institute of Technology, Pasadena, Calif., 2000).

25. D. Z. Anderson, J. C. Frisch, and C. S. Masser, "Mirror reflectometer based on optical cavity decay time," Appl. Opt. 23, 1238-1245 (1984).

26. R. E. Spero, "In situ measurement of cavity parameters needed for calculating shot noise sensitivity," LIGO internal document LIGO-T940068-00-D (California Institute of Technology, Pasadena, Calif., 1994).

27. Others have observed that illuminating the entire surface of a photodiode can cause such an effect, which can be eliminated if only the active region is illuminated (D. H. Shoemaker, Massachusetts Institute of Technology, Cambridge, Mass., personal communication, 1999.) In our case the laser beam illumination was well within the active region whereas the incandescent light illuminated the entire photodiode. Unfortunately we did not try changing the collimation of the incandescent light.

28. Ref. 23, Eq. (10-17), p. 291.

29. Ref. 23, Eq. (10-95), p. 313-314. 